\title{
ELECTROPHYSIOLOGY
}

\section{A new approach for large animal electrophysiology}

\author{
Ulyanova, A.V. et al. eNeuro https://doi.org/10.1523/ENEURO.0102-18.2018 (2018)
}

Humans have gyrencephalic brains. That is, the human brain is folded, with considerable amounts of white matter that transmit the electrical impulses between neurons in the outer cortex and down into subcortical structures like the hippocampus. The folding is thought to be the product of evolutionas mammals like humans evolved and needed more brain power to cope with increasingly complex behavior and social communication, they needed more neurons and cortical connections. But the skull gets in the way and limits how big the brain can grow, so over time the organ began to fold instead.

Rodents, meanwhile, have remained lissencephalic: their brains are smooth and flat, with fewer folds. The rodent cortex lays quite snuggly against the hippocampus, with little white matter in between. The animals are valuable for studying the basics of how neurons communicate, but the relative simplicity of their brains can be a limitation to using rodents to model diseases that are white matter-centric, says John Wolf, a neuroscientist at the University of Pennsylvania and investigator with the Department of Veterans Affairs. Wolf studies traumatic brain injury (TBI) and post-traumatic epilepsy, conditions that can be the result of events like concussions. If there's not a lot of white matter to begin with the biomechanics won't be the same and it can be challenging to translate what's happening in the brain of animal like a rat to that of a human, he explains.

In particular, Wolf and his lab are interested in the hippocampus. This inner region of the brain is responsible for things like cognition and memory, but it's still unclear exactly how the hippocampus communicates with the rest of the brain and how those connections are damaged following an injury. "There's a lot of beautiful, beautiful work in rats that's looking at how spatial encoding is being done, how these spatial aspects are being incorporated with other aspects of cognition," Wolf says; his own career started
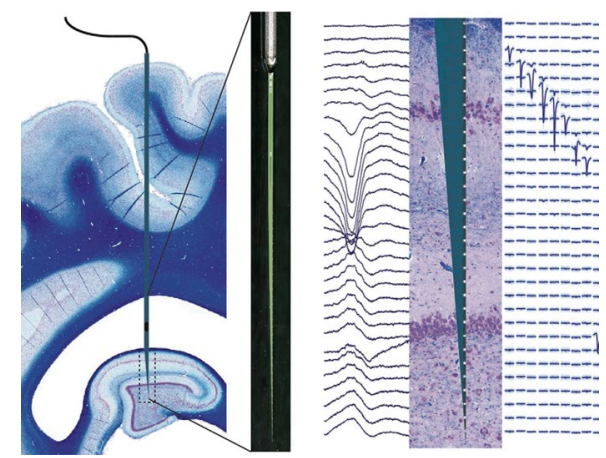

A custom porcine probe for electrophysiology. Credit: Ulyanova, A.V. et al. 2018 (Society for Neuroscience)

with rat neurons. But with an eye towards translation, he and his team have been working with an animal a step closer to the human: the pig.

Given their size and anatomical similarities to people, pigs have historically been an important animal for translational research and for training doctors and surgeons. Wolf is one of a handful of researchers now taking a closer look at their brains, which, like those of humans, are gyrencephalic. His latest publication with first authors Alexandra Ulyanova and Paul Koch and collaborator Kacy Cullen presents an electrophysiological characterization of the hippocampus of the Yucatan miniature pig.

While these particular pigs, developed to be more amenable to the lab, won't reach the 300 pounds typical of their agricultural brethren, the term "minipig" can be a bit of a misnomer to the unfamiliar. They aren't all that mini: the Yucatans can still be over 100 pounds by the time they are fully grown, quite a sizable increase from a rat. Caring for minipigs takes a dedicated animal team, Wolf says, "and there's a huge commitment of resources to doing any surgical work with these animals."

Others have looked at the he hippocampus of the minipig before, in histological samples and with clinical imaging methods like MRI, but Wolf and his coauthors have developed a new in vivo approach that he says could help simplify electrophysiology in swine and reduce the need for expensive imaging equipment. They designed a pig-specific silicon laminar probe, just 80 microns across but with 32 recording channels, to record electrical events through all the layers of the pig hippocampus. To place the customized probe, they adapted deep brain stimulation mapping techniques developed for clinical use in humans. Rather than performing individual MRIs on each animal to create an exact map, the location of the dorsal hippocampus is derived from knowledge of pig anatomy and an electrical map created with smaller electrodes.

Their subsequent electrophysiological recordings and histological samples confirm that the minipig brain sits in an intermediate state between rodents and primates. Features that have been described in rats exist in the pig too, an exciting prospect for translating rodent observations upwards, Wolf says. Electrophysiology is possible in nonhuman primates, but cost ethical considerations are a limiting factor in working extensively in these animals.

They established the proof-of-concept for the method in anesthetized animals, but the brain operates in many states: asleep, awake, moving, resting, Wolf says. To capture that, he and his team are currently working on chronic implants that will enable them to monitor the hippocampus in healthy pigs and those modeling different disease states over longer periods and while the animals are freely behaving.

"That's what's going to allow us to really understand how these diseases are over time progressively affecting the hippocampus," Wolf says.

\section{Ellen P. Neff}

Published online: 23 October 2018 https://doi.org/10.1038/s41684-018-0180-y 